\title{
LABORATORY SIMULATION OF PHYSICAL AND CHEMICAL PROCESSES IN INTERSTELLAR ICES
}

\author{
W.A. SCHUTTE \\ Leiden Observatory \\ P.O. Box 9513, 2300 RA Leiden, The Netherlands
}

\begin{abstract}
We review the techniques which are applied to study by means of laboratory simulation the chemical and physical processes taking place in ices in interstellar dense clouds. We summarize the current situation with regard to spectroscopy of interstellar ice analogs and with regard to the simulation of chemical processes such as modification of the ices by UV photons and surface reactions between reactive atoms and molecules. It is discussed how such data can be used to explore the potential of the icy mantles both as a record of the various chemical conditions that occur in dense clouds and as an environment for unique interstellar chemical processes.
\end{abstract}

\section{Introduction}

Observations of infrared absorption bands superimposed on the continuum emission by obscured embedded and background sources reveal the presence of ices inside dense interstellar clouds (see reviews by Dorschner \& Henning 1995, Schutte 1996, Whittet 1993, 1996). These form when the gas accretes on the cold $(10 \mathrm{~K})$ dust grains. It was early on recognized that the interpretation of such data requires comparison with the spectra of analog ice samples produced in the laboratory (Greenberg 1976, Hagen et al. 1979) Besides to interprete spectroscopy, laboratory simulation has also been applied to study chemical processes that could occur in the icy grain mantles. Such are processing by ultraviolet (UV) radiation, processing by energetic $(\mathrm{MeV})$ particles (protons, helium nuclei), and the chemistry between reactive species at the surface. A number of other processes have been studied in the laboratory, such as photodesorption, thermal sublimation, non-thermal desorption, the sticking efficiency of condensing molecules, the thermal con- 


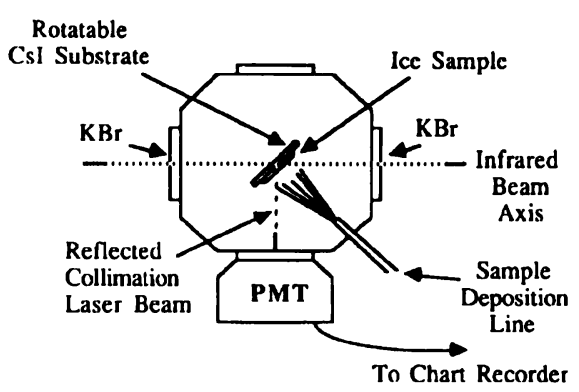

a. Sample Deposition Configuration

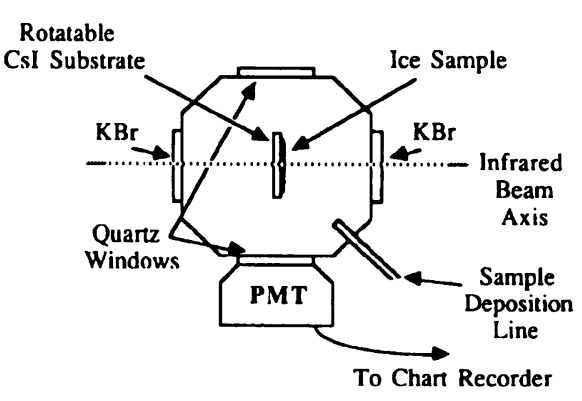

b. Spectrum Collection Configuration

Figure 1. Schematic of the vacuum chamber of a basic set-up to produce interstellar ice analogs. The pictures show the system in the configurations used (a) during sample deposition and (b) during infrared spectral measurement (From Hudgins et al. 1993).

ductivity of amorphous $\mathrm{H}_{2} \mathrm{O}$ ice, and the temperature dependence of the ice structure (Sandford \& Allamandola 1988, Gerakines et al. 1995, Schutte \& Greenberg 1991, Jenniskens \& Blake 1994, Kouchi et al. 1992). However, this overview will be restricted to spectroscopy and chemistry of analogs of interstellar ices.

We will summarize the laboratory techniques that are applied to produce interstellar ice analogs. We review some of the work that has been done and how the results can be used to clarify the composition and evolution of icy grain mantles in dense clouds. Finally, a personal view is given on the nature of future laboratory work most essential for understanding the role that mantles play in the chemistry of dense clouds and protostellar regions.

\section{The production of interstellar ice analogs}

Production of interstellar ice analogs is performed in a vacuum set-up by slow deposition of a mixture of selected gases on a cold substrate. A schematic representation of a basic set-up is given in Fig. 1. Experimental details can for example be found in Hudgins et al. (1993) and Gerakines et al. (1995). Typical experimental conditions are summarized in Table 1 and are compared to conditions in interstellar dense clouds. A sample contamination level of $0.3 \%$ (corresponding to a condensation rate of residual gases in the vacuum of $\sim 3 \times 10^{12} \mathrm{~cm}^{-2} \mathrm{~s}^{-1}$ ) is typical for a set-up with rubber O-ring vacuum seals; for a system with metal sealings an improvement by a factor $10^{2}-10^{4}$ may be obtained.

In the general dense cloud medium the grain temperature is $\sim 10 \mathrm{~K}$ (Greenberg 1971, Mathis et al. 1983, Laureys et al. 1991). Near embedded 
TABLE 1. Interstellar vs. Laboratory conditions

\begin{tabular}{lll}
\hline Parameter & Interstellar & Laboratory \\
\hline Grain Temperature (K) & $\sim 10-70$ & $\geq 10$ \\
Condensation rate (molec. $\mathrm{cm}^{-2} \mathrm{~s}^{-1}$ ) & $10^{5}-10^{7}$ & $\sim 10^{15}$ \\
Condensing species & molec.: $\mathrm{CO}, \mathrm{N}_{2}, \mathrm{O}_{2}, \ldots$ & no problem \\
& atomic: $\mathrm{H}, \mathrm{O}, \mathrm{C}, \ldots$ & generally not \\
UV field (Photons $\left.\mathrm{cm}^{-2} \mathrm{~s}^{-1} ;>6 \mathrm{eV}\right)$ & $10^{3}-10^{8}$ & $10^{15}$ \\
Particle field & $\mathrm{MeV} \mathrm{H}^{+} \& \mathrm{He}^{2+}$ & $\mathrm{KeV} \mathrm{He}^{+}$ \\
\hline
\end{tabular}

protostellar objects, ice temperatures as high as $\sim 100 \mathrm{~K}$ can be observed (Smith et al. 1989). The dense cloud grain temperature may vary by some degrees depending on the depth of the grain in the cloud, the presence of (massive) star formation and the intensity of the local interstellar radiation field. In general such variations should not be an important parameter for the sticking of gaseous molecules, which is believed to be essentially 100 $\%$ even for highly volatile species such as $\mathrm{CO}, \mathrm{O}_{2}$ or $\mathrm{N}_{2}$ (Tielens \& Allamandola 1987). An exception may be $\mathrm{H}_{2}$, which has a small, but significant sticking probability on solid $\mathrm{H}_{2} \mathrm{O}$. With $\mathrm{H}_{2}$ being the paramount species in dense clouds, this may lead to a significant $\mathrm{H}_{2}$ abundance in the ice mantles (Dissly et al. 1994, Buch \& Devlin 1994). The quantity of solid $\mathrm{H}_{2}$ may show considerable dependence on the ice temperature. However, due to the extreme weakness of the infrared transition of the $\mathrm{H}-\mathrm{H}$ stretching mode at $2.415 \mu \mathrm{m}$ (Sandford \& Allamandola 1993), direct observation of such an effect is outside the reach of current astronomical instrumentation.

Direct deposition of atomic and radical species, important for simulating grain surface chemistry, has yet only been performed on a few occasions (see $\S 5)$. Most experiments reported to date have focused on "easy to handle" molecules like $\mathrm{H}_{2} \mathrm{O}, \mathrm{CH}_{3} \mathrm{OH}, \mathrm{NH}_{3}, \mathrm{CO}, \mathrm{O}_{2}$ and $\mathrm{H}_{2} \mathrm{CO}$. With the current disclosure of the entire mid-infrared $(2.5-20 \mu \mathrm{m})$ window by ISO, it is expected that the need for spectroscopy of less stable species will increase (e.g., Hasegawa et al. 1992, 1993).

UV photolysis in the laboratory is generally performed by a hydrogen flow discharge lamp (Hagen et al. 1979, Jenniskens et al. 1993). The bulk of the output lies between $7-10.5 \mathrm{eV}$. To be optically thin in the UV region, the thickness of the ice sample should be at most $\sim 0.1 \mu \mathrm{m}$ (Gerakines et al. 1996). At a typical flux of $10^{15}$ photons $\mathrm{s}^{-1} \mathrm{~cm}^{-2}$, the time between successive absorptions by a molecule is of the order of $10^{3} \mathrm{~s}$, showing that double excitation is negligible. An important pitfall may however involve the UV spectral distribution. In interstellar dense clouds UV radiation is 
expected to be produced either by excitation of $\mathrm{H}_{2}$ by electrons resulting from the ionization of molecular hydrogen by cosmic rays (Sternberg et al. 1987, Gredel et al. 1989), or, in circumstellar regions, in the photospheres of massive stars and the hot inner region of the accretion disk (Spaans et al. 1995). In the former case the spectrum is expected to be rather similar to the lamp spectrum, while in the latter case the interstellar UV spectrum will generally be much softer. We thus emphasize that studying the effects of the applied UV spectral distribution may be important for understanding interstellar photochemistry.

Besides by UV radiation, the icy mantles may also be processed by cosmic rays. While in space this would generally involve hydrogen and helium nuclei in the $\mathrm{MeV}$ energy range, the laboratory simulation has, for practical reasons, been restricted to $\mathrm{KeV}$ energies. While $\mathrm{KeV} \mathrm{He}^{+}$has similar specific energy loss as protons at 1000 times higher energy (Palumbo \& Strazulla 1993), it is unclear whether all aspects of the interaction with the sample are comparable.

No significant temperature rise should occur during irradiation or deposition. Using the heat conduction of amorphous ice $\left(1 \mathrm{erg} \mathrm{cm}{ }^{-1} \mathrm{~s}^{-1} \mathrm{~K}^{-1}\right.$; Kouchi et al. 1992), it is found that at a typical condensation rate of $10^{15}$ molec $\mathrm{cm}^{-2} \mathrm{~s}^{-1}$, the warm-up of the sample is less than $0.1 \mathrm{~K}$. Irradiation with the standard UV flux (Table 1) leads to an increase of at most $1 \mathrm{~K}$.

\section{Infrared spectroscopy of interstellar ice analogs}

The mid-infrared region $(2.5-25 \mu \mathrm{m})$ encompasses the features of the fundamental molecular vibrational modes (stretching, bending, deformation). Spectroscopy of these bands as observed in laboratory analogs of interstellar ice can be directly compared to observations of obscured objects. In this way a number of components have been identified in interstellar ices. The most abundant constituent is $\mathrm{H}_{2} \mathrm{O}$, while $\mathrm{CO}, \mathrm{CO}_{2}$, and $\mathrm{CH}_{3} \mathrm{OH}$ all contribute at the $10 \%$ level relative to $\mathrm{H}_{2} \mathrm{O}$. Furthermore a few percent of $\mathrm{H}_{2} \mathrm{CO}, \mathrm{HCOOH}$ (both still tentative), and $\mathrm{CH}_{4}$ have been found as well as a trace of OCS (See reviews by Schutte 1996, Whittet 1993, Tielens \& Whittet 1997, and furthermore d'Hendecourt \& Jourdain de Muizon 1989, de Graauw et al. 1996, Schutte et al. 1996a,b, Palumbo et al. 1995, Boogert et al. 1996, 1997). Column densities are derived from the equivalent width of the observed feature using the absolute band strength obtained in the laboratory (e.g., Gerakines et al. 1995).

Besides identification and column density, detailed fitting of an observed feature with spectra of the responsible molecule in various ice mixtures may elucidate the nature of the ice in which the molecule is embedded. Such comparison has shown the presence of a separate apolar (likely CO dominated) 


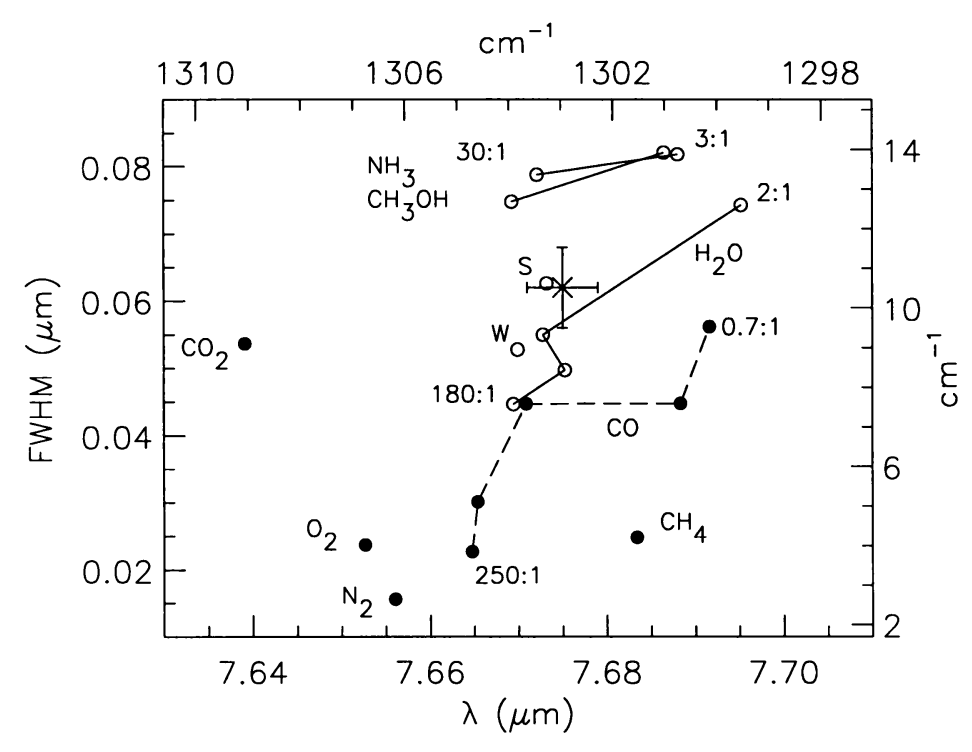

Figure 2. Laboratory spectroscopy of the $\nu_{4}$ mode of interstellar $\mathrm{CH}_{4}$ compared to the observed peak position and FWHM of the solid methane band seen towards NGC7538 IRS9 (cross with error bars). Bullets indicate $\mathrm{CH}_{4}$ in a non-polar matrix, circles a polar matrix. The mixing ratio of $\mathrm{N}_{2}, \mathrm{O}_{2}$, and $\mathrm{CO}_{2}$ with $\mathrm{CH}_{4}$ is $20: 1$. The "S" indicates the mixture $\mathrm{H}_{2} \mathrm{O}: \mathrm{CH}_{3} \mathrm{OH}: \mathrm{CO}: \mathrm{CH}_{4}=70: 40: 1: 1$, while "W" represents 100:10:1:1 (same species). For the other mixtures a line is drawn between points with an increasing $\mathrm{CH}_{4}$ abundance. For each series the highest and lowest abundance relative to $\mathrm{CH}_{4}$ are indicated. The temperature for all mixtures is $10 \mathrm{~K}$ (from Boogert et al. 1996).

ice phase next to the dominant solid $\mathrm{H}_{2} \mathrm{O}$ (Sandford et al. 1988, Tielens et al. 1991). Furthermore fitting the $9.75 \mu \mathrm{m}$ feature of solid $\mathrm{CH}_{3} \mathrm{OH}$ points to the existence of a component enriched in methanol (Skinner et al. 1992). Similar analysis of the features observed for various molecules may clarify how the ice components are distributed over these different phases. This would evidently be of great importance for assessing the chemical conditions which may exist in dense clouds throughout space and time. Spectroscopic databases are now available for $\mathrm{H}_{2} \mathrm{O}$ (Ehrenfreund et al. 1996a), $\mathrm{CO}, \mathrm{CO}_{2}$, $\mathrm{CH}_{4}, \mathrm{CH}_{3} \mathrm{OH}, \mathrm{H}_{2} \mathrm{CO}$ and $\mathrm{SO}_{2}$, and can be obtained through the world wide web (for $\mathrm{CO}$ and $\mathrm{CO}_{2}$; Ehrenfreund et al. 1996b) or upon request from the author (for $\mathrm{H}_{2} \mathrm{O}, \mathrm{CH}_{4}, \mathrm{CH}_{3} \mathrm{OH}, \mathrm{H}_{2} \mathrm{CO}$, and $\mathrm{SO}_{2}$ ).

Figure 2 shows an example of how the profile and position of a feature (in this case the $\nu_{4}$ mode of $\mathrm{CH}_{4}$ near $7.67 \mu \mathrm{m}$ ) can vary with ice composition (Boogert et al. 1996). A good overview of the behaviour is obtained in this diagram which shows the width vs. wavelength in various matrices. 
The comparison with the solid $\mathrm{CH}_{4}$ feature towards the obscured protostar NGC7538 IRS9 shows that the interstellar $\mathrm{CH}_{4}$ ice is frozen in a polar environment, either $\mathrm{H}_{2} \mathrm{O}$ dominated or $\mathrm{H}_{2} \mathrm{O} / \mathrm{CH}_{3} \mathrm{OH}$ dominated.

\section{Energetic processing of interstellar ice analogs}

The effects of energetic processing of interstellar ice analogs have been studied both for UV irradiation and for processing by energetic ions, although the results on ion processing of astronomically relevant ices has so far been somewhat limited (see overview Schutte 1996). The role of energetic processing in the chemical evolution of, in particular, protostellar regions and the pre-solar nebula is one of the most important issues of interstellar chemistry. Laboratory data can be used in analyzing observations of solid state and gaseous interstellar molecules to search for the molecular fingerprints of processing.

First, monitoring by infrared spectroscopy of the modification can be compared to the spectra of interstellar ices. Infrared spectroscopy primarily probes relatively simple species which are most abundantly formed by irradiation. Examples of such species produced by UV photolysis are $\mathrm{CO}_{2}$ (from $\mathrm{CO}$ and $\mathrm{H}_{2} \mathrm{O}$, or $\mathrm{CO}$ and $\mathrm{O}_{2}$ ), $\mathrm{HCOOH}$ (from $\mathrm{CO}$ and $\mathrm{H}_{2} \mathrm{O}$ ), $\mathrm{HCONH}_{2}$ (from $\mathrm{CO}$ and $\mathrm{NH}_{3}$ ), $\mathrm{H}_{2} \mathrm{CO}$ (from $\mathrm{CH}_{3} \mathrm{OH}$, or $\mathrm{H}_{2} \mathrm{O}$ with $\mathrm{CO}$ ) and $\mathrm{CH}_{4}$ (from $\mathrm{CH}_{3} \mathrm{OH}$ ) (d'Hendecourt et al. 1986, Gerakines et al. 1996, Allamandola et al. 1988). Furthermore, some simple ions such as $\mathrm{OCN}^{-}$and $\mathrm{HCOO}^{-}$are formed (Grim \& Greenberg 1987, Grim et al. 1989)

Second, the processing of interstellar ice analogs generally leads to the formation of a non-volatile organic residue (Agarwal et al. 1985, Briggs et al. 1992, Bernstein et al. 1995, Moore et al. 1983, Strazzulla \& Baratta 1992). Detailed analysis by Gas Chromatography/Mass Spectroscopy (GC/MS) was performed for the residues obtained by UV photolysis of $\mathrm{H}_{2} \mathrm{O} / \mathrm{CO} / \mathrm{NH}_{3}$ (Agarwal et al. 1985, Briggs et al. 1992) and of $\mathrm{H}_{2} \mathrm{O} / \mathrm{CH}_{3} \mathrm{OH} / \mathrm{NH}_{3}$ (Bernstein et al. 1995). The results showed that they consist of a mixture of complex, O-rich organic species, and are generally dominated by molecules derived from formaldehyde polymers (Schutte 1988, Bernstein et al. 1995). This is not surprising in view of the great reactivity of $\mathrm{H}_{2} \mathrm{CO}$ even at cryogenic temperatures (Schutte et al. 1993). Since the residues generally only make up at most a few percent of the mass of the original ice (Jenniskens et al. 1993, Bernstein et al. 1995), the chance of remote observing these components in interstellar dense clouds seems, indeed, remote. However, such products could be preserved in comets insofar these represent pristine pre-solar nebula material. Indeed, it was proposed that they could be responsible for (part of) the abundant organic component of comets (Greenberg 1982, Mumma et al. 1993). 
Finally, mm surveys of the rotational transitions of gas phase species towards hot cores probe the composition of the ice mantles which sublime in such regions (Millar 1997). The advantage of such data over direct infrared spectroscopic inventarisation of the ices is a much greater sensitivity. In principle, $\mathrm{mm}$ observations may detect ice components down to ppm abundances. This makes this technique especially suitable to search for the molecular fingerprints of energetic processing. The natural laboratory counterpart would be the analysis of the subliming sample by means of GC/MS or mass spectroscopy. Up to now, only very few data of this kind exist (Bernstein et al. 1995) and more extensive laboratory surveys are highly desirable.

\section{Simulation of grain surface processes}

Grain surface processes are supposed to play a dominant role in shaping the composition of interstellar ices. It is considered that such processes transform the highly reactive species (atoms, radicals) which are abundant in the gas phase into the relatively stable molecules in the icy mantles. For example, the most abundant mantle component, $\mathrm{H}_{2} \mathrm{O}$, is supposed to primarily form by surface reactions of atomic oxygen and hydrogen (Tielens \& Hagen 1982). Clearly, knowledge of grain surface chemistry is of paramount importance to understand the origin of the icy molecules and the relationship between the composition of the mantles and the chemical conditions in the gas phase at the time of accretion. Unfortunately, experimental simulation in this field is still very rare. Recently, studies have been published on the reaction $\mathrm{N}+3 \mathrm{H} \rightarrow \mathrm{NH}_{3}$ and on the formation of $\mathrm{H}_{2} \mathrm{CO}$ and $\mathrm{CH}_{3} \mathrm{OH}$ from $\mathrm{CO}$ and atomic $\mathrm{H}$ (Hiraoka et al. 1994, Hiraoka et al. 1995). However, many important reactions such as $\mathrm{CO}+\mathrm{O} \rightarrow \mathrm{CO}_{2}, \mathrm{H}_{2} \mathrm{O}_{2}+\mathrm{H} \rightarrow \mathrm{H}_{2} \mathrm{O}+\mathrm{OH}$ and the hydrogenation of $\mathrm{CO}_{2}$ and $\mathrm{N}_{2}$ still need investigation.

\section{The future}

It is expected that laboratory simulation of the physical and chemical processes taking place in and on icy grain mantles will continue to play an essential role in the study of dense clouds and protostellar regions. We feel that a number of laboratory studies could be of special significance. First, in the near future analyzing the ice spectroscopy by ISO over the entire mid-infrared range $(2.5-25 \mu \mathrm{m})$ will require infrared studies of new compounds. Molecules which one can reasonably expect to find are a.o. HCN, $\mathrm{C}_{2} \mathrm{H}_{2}, \mathrm{H}_{2} \mathrm{O}_{2}, \mathrm{O}_{3}, \mathrm{HOCN}, \mathrm{HNCO}, \mathrm{H}_{2} \mathrm{CO}_{3}, \mathrm{~N}_{2} \mathrm{H}_{2}$, and $\mathrm{N}_{2} \mathrm{H}_{4}$ (e.g., Hasegawa et al. 1992, 1993). Such measurements would have to involve dilution in po$\operatorname{lar}\left(\mathrm{H}_{2} \mathrm{O}, \mathrm{CH}_{3} \mathrm{OH}\right)$ as well as apolar ices $\left(\mathrm{CO}, \mathrm{O}_{2}\right)$. 
A project of major importance would be a detailed survey of the products of energetic processing of the ice analogs. This requires GC/MS analysis of volatile products as well as of the refractory organic residue. Such a study may encompass a variety of starting ice mixtures of relevant species (e.g., §3). Special emphasis should be placed on analyzing the difference between UV and energetic ion processing. The resulting database would be of great value for the interpretation of $\mathrm{mm}$ data of hot cores as well as of the results of ROSETTA's mission to comet P/Wirtanen.

Finally, grain surface chemistry, so vital to establish the link between gas phase chemistry and icy mantle composition, will need to be thoroughly studied in the laboratory. Indeed, until such studies have been performed the origin of some of the most important components of interstellar ices, e.g., $\mathrm{CH}_{3} \mathrm{OH}$ and $\mathrm{CO}_{2}$, remains elusive.

\section{Acknowledgements}

We thank Adwin Boogert for making the diagram on $\mathrm{CH}_{4}$ spectroscopy available. David Jansen's assistance with the layout of this manuscript was a great support.

\section{References}

Agarwal, V.K., Schutte, W.A., Greenberg, J.M., et al. 1985, Origins of Life 16, 21

Allamandola, L.J., Sandford, S.A., Valero, G.J. 1988, Icarus 76, 225

Bernstein, M.P., Sandford, S.A., Allamandola, L.J., Chang, S., Scharberg, M.A., 1995, ApJ 454, 327

Boogert, A.C.A., Schutte, W.A., Tielens, A.G.G.M., Whittet, D.C.B., Helmich, F.P., Ehrenfreund, P., Wesselius, P.R., de Graauw, Th., Prusti, T. 1996, A\&A, 315, L377

Boogert, A.C.A., Schutte, W.A., Helmich, F.P., Tielens, A.G.G.M., Wooden, D.H. 1997, A\&A, in press

Briggs, R., Ertem, G., Ferris, J.P., et al. 1992, Origins of Life and Evolution of the Biosphere 22, 287

Buch, V., Devlin, J.P. 1994, ApJ 431, L135

de Graauw, Th., Whittet, D.C.B., Gerakines, P.A., et al. 1996, A\&A, 315, L345

d'Hendecourt, L.B., Allamandola, L.J., Grim, R.J.A., Greenberg, J.M. 1986, A\&A 158, 119

d'Hendecourt, L.B., Jourdain de Muizon, M. 1989, A\&A 223, L5

Dissly, R.W., Allen, M., Anicich, V.G. 1994, ApJ 435, 685

Dorschner, J., Henning, T. 1995, A\&AR 6, 271

Ehrenfreund, P., Gerakines, P.A., Schutte, W.A., van Hemert, M., van Dishoeck, E.F. 1996a, A\&A, 312, 263

Ehrenfreund, P., Boogert, A.C.A., Gerakines, P.A., Jansen, D.J., Schutte, W.A., Tielens, A.G.G.M., and van Dishoeck, E.F. 1996b, A\&A, 315, L341

Gerakines, P.A., Schutte, W.A., Greenberg, J.M., van Dishoeck, E.F. 1995, A\&A 296, 810

Gerakines, P.A., Schutte, W.A., Ehrenfreund, P.A. 1996, A\&A, 312, 289

Gredel, R., Lepp, S., Dalgarno, A., Herbst, E. 1989, ApJ 347, 289

Greenberg, J.M. 1971, A\&A 12, 240

Greenberg, J.M. 1976, Ned. Tijdschr. Natuurkd. 42, 117 
Greenberg, J.M. 1982, in Comets, ed. L.L. Wilkening (Tucson, University of Arizona press), p. 131

Grim, R.J.A., Greenberg, J.M. 1987, ApJ 321, L91

Grim, R.J.A., Greenberg, J.M., de Groot, M.S., Baas, F., Schutte, W.A., Schmitt, B. 1989, A\&AS 78, 161

Hagen, W., Allamandola, L.J., Greenberg, J.M. 1979, Ap\&SS 65, 215

Hasegawa, T.I., Herbst, E., Leung, C.M. 1992, ApJS 82, 167

Hasegawa, T.I., Herbst, E. 1993, MNRAS 261, 83

Hiraoka, K., Ohashi, N., Kihare, Y., Yamamoto, K., Sato, T., Yamashita, A. 1994, Chemical Physics Letters 229, 408

Hiraoka, K., Yamashita, A., Yachi, Y., Aruga, K., Sato, T. 1995, ApJ 443, 363

Hudgins, D.M., Sandford, S.A., Allamandola, L.J., Tielens, A.G.G.M. 1993, ApJS 86, 713

Jenniskens, P., Baratta, G.A., Kouchi, A., de Groot, M.S., Greenberg, J.M., Strazulla, G. 1993, A\&A 273, 583

Jenniskens, P., Blake, D.F. 1994, Science 265, 709

Kouchi, A., Greenberg, J.M., Yamamoto, T., Mukai T. 1992, ApJ 388, L73

Laureys, R.J., Clark, F.O., Prusti, T. 1991, ApJ 372, 185

Mathis, J.S., Mezger, P.G., Panagia, N. 1983, A\&A 128, 212

Millar, T.J. 1997, this volume

Moore, M.H., Donn, B., Khanna, R., A'Hearn, M.F. 1983, Icarus 54, 388

Mumma, M.J., Weissman, P.R., Stern, S.A. 1993, in Protostars and Planets III, eds. E.H. Levi \& J.I. Lunine (Tucson, University of Arizona Press), p. 1177

Palumbo, M.E., Strazulla, G. 1993, A\&A 269, 568

Palumbo, M.E., Tielens, A.G.G.M., Tokunaga, A.T. 1995, ApJ 449, 674

Sandford, S.A., Allamandola, L.J. 1988, Icarus 76, 201

Sandford, S.A., Allamandola, L.J., Tielens, A.G.G.M., Valero, L.J. 1988, ApJ 329, 498

Sandford, S.A., Allamandola, L.J. 1993, ApJ 417, 815

Schutte, W.A. 1988, PhD thesis, University of Leiden, Leiden

Schutte, W.A., Greenberg, J.M. 1991, A\&A 244, 190

Schutte, W.A., Allamandola, L.J., Sandford, S.A. 1993, Icarus 104, 118

Schutte, W.A. 1996, in The Cosmic Dust Connection, ed. J. M. Greenberg (Kluwer, Dordrecht), in press

Schutte, W.A., Gerakines, P.A., Geballe, T.R., van Dishoeck, E.F., Greenberg, J.M. 1996a, A\&A 309, 633

Schutte, W.A., Tielens, A.G.G.M., Whittet, D.C.B., Boogert, A., Ehrenfreund, P., de Graauw, Th., Prusti, P., van Dishoeck, E.F., Wesselius, P. 1996b, A\&A, 315, L333

Skinner, C.J., Tielens, A.G.G.M., Barlow, M.J., Justtanont, K. 1992, ApJ 399, L79

Smith, R.G., Sellgren, K., Tokunaga, A.T. 1989, ApJ 344, 413

Spaans, M., Hogerheijde, M.R., Mundy, L.G., van Dishoeck, E.F. 1995, ApJ 455, L167

Sternberg, A., Dalgarno, A., Lepp, S. 1987, ApJ 320, 676

Strazzulla, G., Baratta, G.A. 1992, A\&A 266, 434

Tielens, A.G.G.M., Hagen, W. 1982, A\&A 114, 245

Tielens, A.G.G.M., Allamandola, L.J. 1987, in Interstellar Processes, eds. D.J. Hollenbach \& H.A. Thronson (Reidel, Dordrecht), p. 397

Tielens, A.G.G.M., Tokunaga, A.T., Geballe, T.R., Baas, F. 1991, ApJ 381, 181

Tielens, A.G.G.M., Whittet, D.C.B. 1997, this volume

Whittet, D.C.B. 1993, in Dust and Chemistry in Astronomy, eds. T.J. Millar \& D.A. Williams (Cambridge University Press), p. 9

Whittet, D.C.B. 1996, in The Cosmic Dust Connection, ed. J. M. Greenberg (Kluwer, Dordrecht), in press 


\section{Discussion}

Herbst: What is the status of the grain surface reacion $\mathrm{CO}+\mathrm{O} \rightarrow \mathrm{CO}_{2}$ ?

Schutte: Early work seemed to indicate that this reaction does not proceed under cryogenic conditions (Grim \& d'Hendecourt 1986, A\&A 167, 161). However, in these experiments the atomic $\mathrm{O}$ was produced by photolysis of $\mathrm{O}_{2}$. This species was still abundant during the subsequent warm-up leading to efficient formation of $\mathrm{O}_{3}$ due to the reaction of molecular oxygen with atomic oxygen. While little $\mathrm{CO}_{2}$ production was observed under these conditions, it cannot yet be concluded that the $\mathrm{CO}+\mathrm{O}$ reaction does proceed in the absence of a large $\mathrm{O}_{2}$ abundance.

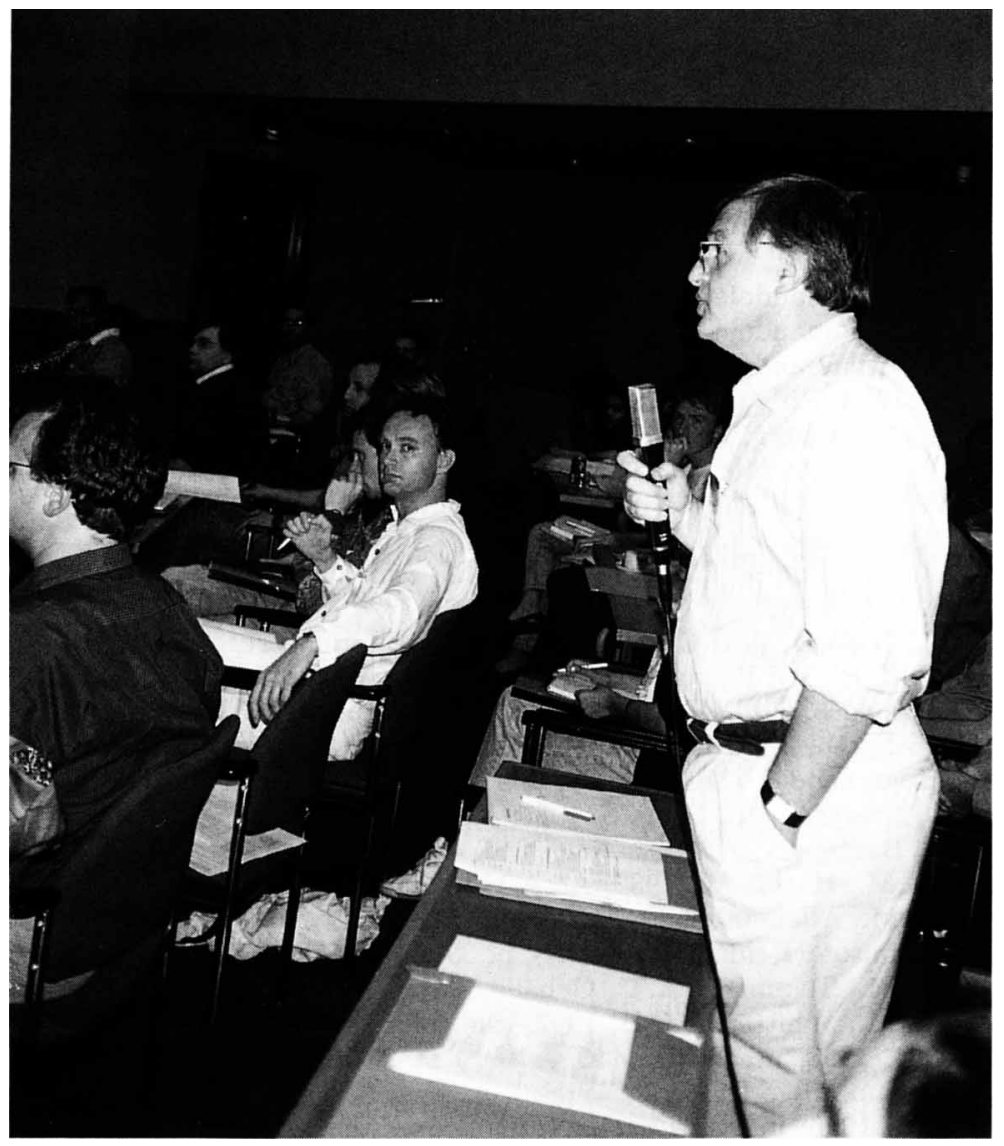

